\title{
Problems in Geometric Probing ${ }^{1}$
}

\author{
Steven S. Skiena \\ Department of Computer Science \\ State University of New York \\ Stony Brook, NY 11794
}

February 7, 1989

\begin{abstract}
Many sensing problems in robotics can be formulated as problems in geometric probing; that is, given geometric models of a sensor and an object, what information can be determined about the object and how efficiently can it be found. We review results in geometric probing and give a collection of open problems for a variety of probing models and objects.
\end{abstract}

Keywords: Geometric probing, theory of robotics, computational geometry, discrete geometry, finger probes, half-planes, $\mathrm{x}$-rays, tomography, convexity, complexity, graph theory.

${ }^{1}$ This research was partially supported by National Science Foundation Grant CCSR-8714565 and a University of Illinois Summer Fellowship in Computer Science. 


\section{Introduction}

Geometric probing considers problems of determining a geometric structure or some aspect of that structure from the results of a mathematical or physical measuring device, a probe. A variety of problems from robotics, medical instrumentation, mathematical optimization, integral and computational geometry, graph theory and other areas fit into this paradigm. These problems are interesting in themselves, but the results also have application to these and other fields. This paper surveys the work in geometric probing, emphasising interesting open problems.

The problem of geometric probing was introduced by Cole and Yap [1] and inspired by work in robotics and tactile sensing [23435678]. It has since inspired no less than three dissertations [91011] and several additional papers [121314]. The most complete description of these results appear in [10].

In this paper, we describe a taxonomy of probing problems, their history, and open problems for each probing model. A vast number of probing problems can be defined, since we can take a "Chinese menu" approach to generating them. Choosing from column $A$, there are a wide variety of interesting models of sensors, with inspiration either from physical sensing devices or geometrical operations. Our main probing models:

(1) Finger Probes - which measure the first point of intersection between a directed line and an object.

(2) Hyperplane Probes - which measure the first time when a hyperplane moving parallel to itself intersects an object.

(3) X-ray Probes - which measure the length of intersection between a line and an object.

(4) Half-space Probes - which return the area or volume of intersection between a half-space and an object.

(5) Cut-set Probes - which for a specified graph and partition of the vertices returns the size of the cut-set represented by the partition.

More sophisticated sensing devices can be constructed by considering aggregates of probes sharing certain properties. For example, the set of all probes which are parallel to a given line or which pass through a given point. We can also consider the power gained by having access to more than one type of probe. How well do sensors work together to determine objects?

In column $B$ of our menu, we have constraints on the type of object being probed. Most results hold only for convex polygons. For certain probing models, extensions to more general objects are impossible. Other objects of interest include collections of convex polygons, star-shaped and simple polygons, point sets, straight line graphs, polytopes in three or higher dimensions, and continuous surfaces of specified degree. In all cases, we can also consider restricting the objects to come from a known finite class to create model-based problems. Interesting problems also arise when the disparity in dimension between object and probe increase beyond one.

Finally, in column $C$ is the property which we are interested in optimizing or bounding:

(1) Determination - how many probes are necessary to completely determine a particular object. We are interested in both upper and lower bounds.

(2) Verification - given a reputed description of the object how many probes are necessary to test if the description is valid?

(3) Computational Complexity - the complexity of planning the probes can be measured under a RAM cost model, assuming that an oracle returns the results for a specified probe in unit time.

(4) Simulation - given a probe model and a representation of the object, how much time and space is necessary to simulate an actual probe?

(5) Feature Determination - how many probes are needed to determine some feature of the object, such as volume, orientation, or convexity?

Results in probing are most naturally ordered by probing model. For each model, we summarize what is known and present open problems which appear interesting. 


\section{Finger Probes}

A finger probe is defined as the first point of intersection between a directed line $l$ and an object $P$. In the first paper in the area of geometric probing, Cole and Yap [1] give a finger probing strategy for convex polygons in the plane requiring at most $3 n$ probes. This strategy involves two phases, the first of which terminates when three probe contact points are incident upon the same line and hence the same edge. The second phase walks around the polgon and repeatedly aims at the conjectured vertex defined by the known edge and the next two probes. This strategy insures that, in general, at most two probes are incident upon the relative interior of each edge and one probe contacts each vertex to give the $3 n$ bound. They also give a proof that $3 n-1$ probes are necessary, which improves to $3 n$ under a mild assumption. Their strategy can also be shown to have $O(n)$ time complexity, making it optimal for two-dimensional finger probing. It is easily seen that $2 n$ finger probes are necessary and sufficient to verify a convex polygon in two dimensions, by contacting each vertex and the interior of each edge once.

(1) Tighten the gap between the lower and upper bounds for determination in higher dimensions. Dobkin, Edelsbrunner, and Yap [15] consider finger probing in higher dimensions, proving that $f_{0}(P)+f_{d-1}(P)$ probes are necessary for determination of a convex $d$-polytope $P$ and that $f_{0}(P)+(d+2) f_{d-1}(P)$ probes are sufficient, where $f_{i}(P)$ denotes the number of $i$-dimensional faces of $P$. For $d=3$, the lower bound has been raised to $f_{0}(P)+2 f_{2}(P)[10]$.

(2) What is the time complexity of finger probing in higher dimensions?

(3) How many probes are necessary to verify or determine a convex $n$-gon when $n$ is known? We have shown [10] that $3\lceil n / 2\rceil$ probes are sufficient to verify and $3 n-1$ probes sufficient to determine $P$ when $n$ is known, although we do not have interesting lower bounds.

\subsection{Hyperplane Probes}

Dobkin, Edelsbrunner, and Yap [15] define a hyperplane probe to be the time of intersection between a hyperplane $H$, approaching from infinity in the direction of its normal, and the object $P$. They use a duality argument to prove that finger probes through the origin are identical in power to hyperplane probes, independently developed by Greschak [9]. Li [12] provides a proof that $3 n+1$ line probes in $E^{2}$ are both necessary and sufficient to determine convex polygons, which illustrates that only certain finger probing results dualize to hyperplane probes. Li [ 12] also defines a projection probe as two hyperplane probes of identical slope moving from both directions. He shows that $3 n-2$ such probes are both necessary and sufficient for determination. Weaker bounds for this problem were independently discovered by Narasimhan [11].

(4) Bernstein [13] considered the problem of identifying a convex polygon $P$ from a given set of $m$ such convex polygons, and shows that $2 n+k$ finger probes are sufficient, where $k$ is a small constant independent of $m$ based on various assumptions of what is known about $P$. Bernstein's model based finger probing strategy does not dualize to hyperplane probes. Is there a better than $3 n+1$ strategy for hyperplane probing one of a known finite set of convex $n$-gons?

(5) Projection probes are two-dimensional versions of silhouette probes, which return the shadow cast by a polytope in a specified direction. These dualize to cross-section probes which return a slice of the polytope. Dobkin, Edelsbrunner, and Yap [15] show that $f_{0}(P) / 2$ cross-section probes are necessary and $f_{0}(P)+5 f_{2}(P)$ are sufficient to determine a convex polyhedron in $E^{3}$. These bounds dualize to silhouettes by reversing the role of $f_{0}$ and $f_{2}$. Tighten the bounds on determination with silhouettes in $E^{3}$. 


\subsection{X-ray Probes}

X-ray probes represent a discrete model of the sensing devices used in tomography [16] and other forms of medical instrumentation. Edelsbrunner and Skiena [17] define an x-ray probe as measuring the length of intersection between a line and polytope $P$. We have shown that $2 n$ x-ray probes are necessary to determine a convex $n$-gon using a topological argument and give a strategy for determining a convex $n$-gon in $5 n+19$ xray probes. This strategy is based on the observation that four $\mathrm{x}$-ray probes through a common point which pass through the same two edges are in general sufficient to reconstruct the edges. Three parallel probes are used to confirm that these probes contact the same edges. Once the first edge has been determined, Cole and Yap's finger probing strategy can be simulated to determine the rest. However, there are many subtleties in this strategy, enough so that the result is probably not tight.

(6) Tighten the gap between our lower and upper bounds for determination. We conjecture that $3 n+c$ probes are necessary and sufficient, since up to $2 n$ probes in our strategy are "wasted" locating the first edge pair.

(7) We show that $n$ x-ray probes are necessary and $3 n / 2+6$ sufficient for verification of convex $n$-gons. Tighten the gap between these upper and lower bounds.

(8) Can the techniques of x-ray probes be applied to real tomographic systems? Specifically, how effective are algebraic reconstruction techniques [16] when probing directions can be interactively selected?

\subsection{Half-Space Probes}

According to legend, Archimedes determined the authenticity of his king's crown by dunking it in a tub and measuring the volume of water displaced. We [18] have considered the problem of half-plane probing, where a half-plane probe returns the area of intersection between a closed half-plane and an object $P$, and give a strategy for determining a convex $n$-gon in $7 n+7$ half-plane probes, with a lower bound of $2 n$ probes. The structure of this strategy is based on techniques developed for x-ray probing, since we can exploit the integral/differential relationship between parallel x-ray and half-plane probes. Also, we give an $n+1$ verification strategy, with $n / 2$ probes as a lower bound for verification.

(9) Tighten determination and verification bounds for half-planes. Again, we conjecture that $3 n+c$ probes are necessary and sufficient.

(10) The obvious generalizations to higher dimensions appear difficult. A half-space probe returns the volume of intersection between a polytope $P$ and the half-space. A cross-sectional area probe returns the area of intersection between a plane and $P$. Is there a finite strategy for reconstructing convex polyhedra in $E^{3}$ from halfspace or cross-sectional area probes?

(11) Since each half-plane probe can be considered as a function of the entirety of $P$, it is reasonable to consider generalizing to a wider class of objects. Does there exist a finite probing strategy for reconstructing star-shaped polygons from half-plane probes? Such a strategy cannot exist for X-ray probes.

\subsection{Aggregate Probes}

For several of the probing models, considering the complete set of probes sharing a particular feature as one measurement provides a great increase in power. For example, x-ray pictures, which return the entire histogram of a given direction, permit the possibility of extending probing results to general polygons. This is because each probe measures some aspect of the entire polygon, thus eliminating the trivial objection that some small crack will not be found in a finite number of probes. Such problems have been studied from several different perspectives. 
P. C. Hammer [19] posed the following problems in 1963: How many x-ray pictures must be taken to permit exact reconstruction of a convex body if the x-rays issue from a finite point source? How many are needed if the x-rays are assumed parallel? These problems have since generated a substantial literature [2021222324] which is based on integral geometry. The distinction between the two problems is exactly the distinction between origin and parallel probing models. We can also distinguish between oblivious and non-oblivious strategies, where oblivious strategies do not consider the results of previous probes in formulating the next probe.

Gardner [23] shows that three parallel x-ray pictures are necessary and sufficient to verify a convex set and Edelsbrunner and Skiena [17] that three x-ray pictures are necessary and sufficient to determine a convex polygon. Gardner and McMullen [20] prove that four oblivious parallel x-ray pictures are sufficient to determine any convex set. Most of these results are derived for convex sets, not the more restricted case of convex polygons. However, since a convex set can be approximated arbitrarily closely by a convex polygon, many results for polygons should also hold for convex sets. Simpler proofs of these results follow for convex polygons.

(12) How many x-ray photograph probes are necessary to determine a star-shaped $n$-gon? This generalizes Hammer's problems beyond convex polygons. The biggest problem in extending probing results to star-shaped polygons is the existence of invisible vertices, structures which make it difficult to prove that a section of $P$ contains no vertices. For general polygons the histogram no longer contains a vertex corresponding to each vertex of the polygon. It is a problem of combinatorial interest how many such projections there can be in a point set. A related problem is that of the number of $k$-projections in a point set $N$, where a $k$-projection is a direction $l$ such that $d \leq k$ points of $N$ project onto $l[25]$.

(13) A different notion of parallelism involves determining $P$ when we can make $k$ distinct probes in a round. How many rounds of $k$ finger probes per round are necessary to determine a convex $n$-gon? We have shown [10] that $8 n / 3$ rounds are sufficient when $k=2$, but have no interesting results for $k \geq 3$.

(14) We can generalize probing strategies beyond homogeneous probes. Interesting problems result when we have access to more than one type of probing device. Greschak [9] asks how many probes are necessary to determine a convex polygon $P$ when we can use both finger and hyperplane probes?

(15) How many probes are required for determination given access to both finger and x-ray probes. Clearly $3 n$ is a lower bound but does access to the $\mathrm{x}$-ray probe help?

\subsection{Cut-set Probes}

Mathematial probes with little or no physical interpretation give interesting problems. We define a cut-set probe to be a straight line which partitions the vertices of a graph embedded in the plane into two subsets and returns the size of the cut-set defined by the partition. We have shown [26] that $\left(\begin{array}{l}n \\ 2\end{array}\right)$ cut-set probes are sufficient to determine a straight line graph whose vertices are in general position. Further, this bound is tight for verification and determination when $n$ is even. If we remove the geometric restriction and can inquire about the size of an arbitrary cut-set, $\Theta\left(n^{2} / \log n\right)$ probes are necessary and sufficient for reconstruction.

(16) Are fewer probes needed to determine the number of edges in a graph? How about the degree sequence of $G$ ?

(17) Are fewer probes necessary for special types of graphs, such as planar graph embeddings? $O(n \log n)$ arbitrary cut-set probes are sufficient to determine a tree, while lower bounds can be based on enumeration results [27] for the class of graph. 


\section{Conclusions}

Geometric probing is a new and largely unexplored area of computational geometry. We have presented the state of the art in probing research and given interesting open problems in several areas. Further research in this area can lead to insights into fields diverse as medical instrumentation and mathematical optimization and we hope to lay the foundations for such work.

\section{Acknowledgements}

I would like to thank Herbert Edelsbrunner for calling my attention to the problem of probing and his exceedingly patient advice and teaching. Discussions with S. Y. Bob Li have improved my understanding of lower bounds for probing and introduced new problems. Vasant Rao posed the problem of X-ray probing, Nate Dean suggested cut-set probes, and Prabhakar Raghavan the problem of probing in rounds. 


\section{References}

\section{References}

1. Richard Cole and Chee K. Yap, "Shape from Probing," Journal of Algorithms 8(1) pp. 19-38 (1987).

2. Jacob T. Schwartz and Micha Sharir, Some Remarks on Robot Vision, New York University, Courant Institute of Mathematical Sciences, New York (April 1984).

3. Peter C. Gaston and Tomas Lozano-Perez, "Tactile Recognition and Localization Using Object Models: The Case of Polyhedra on a Plane," IEEE Transactions on Pattern Analysis and Machine Intelligence PAMI-6 pp. 257-266 (May 1984).

4. W. Eric L. Grimson and Tomas Lozano-Perez, "Model-Based Recognition and Localization from Sparse Range or Tactile Data," International Journal of Robotics Research 3 pp. 3-35 (Fall 1984).

5. W. Eric L. Grimson and Tomas Lozano-Perez, "Localizing Overlapping Parts by Searching the Interpretation Tree," IEEE Transactions on Pattern Recognition and Machine Intelligence PAMI-9 pp. 469-482 (July 1987).

6. R. E. Ellis, Edward M. Riseman, and Allen R. Hanson, "Tactile Recognition by Probing: Identifying a Polygon on a Plane," Proc. Amer. Assoc. of Art. Intelligence Conf., pp. $632-637$ (1986).

7. W. Eric L. Grimson, "Sensing Strategies for Disambiguating Among Multiple Objects in Known Poses," IEEE Journal of Robotics and Automation RA-2 pp. 196-213 (December 1986).

8. W. Eric L. Grimson, "The Combinatorics of Local Constraints in Model-Based Recognition and Localization from Sparse Data," J. ACM 33 pp. 658-686 (October 1986).

9. John Paul Greschak, Reconstructing Convex Sets, Ph.D. Dissertation, MIT, Department of Electrical Engineering and Computer Science, Cambridge MA (1985).

10. Steven S. Skiena, Geometric Probing, Doctoral Dissertation, University of Illinois, Department of Computer Science, Urbana, IL (1988).

11. L. S. Narasimhan, Recognition of Polyhedral Objects: Concepts and Algorithms, Doctoral Dissertation, School of Management Science, University of Texas, Dallas TX (1988).

12. S. Y. Robert Li, "Reconstruction of Polygons from Projections," Information Processing Letters $\mathbf{2 8}$ pp. 235-240 (1988).

13. Herbert J. Bernstein, "Determining the Shape of a Convex $n$-sided Polygon by Using $2 n+k$ Tactile Probes," Information Processing Letters 22 pp. 255-260 (1986).

14. B. K. Natarajan, "On Detecting the Orientation of Polygons and Polyhedra," Proc. Third ACM Symposium Computational Geometry 3 pp. 146-152 (1987).

15. David P. Dobkin, Herbert Edelsbrunner, and Chee K. Yap, "Probing Convex Polytopes," Proc. 18th ACM Symposium on the Theory of Computing, pp. 424-432 (1986).

16. Gabor T. Herman, Image Reconstruction from Projections: the Fundamentals of Computerized Tomography, Academic Press, New York (1980).

17. H. Edelsbrunner and S. S. Skiena, "Probing Convex Polygons with X-rays," SIAM J. Computing 17 pp. 870-882 (1988).

18. Steven S. Skiena, Probing Convex Polygons with Half-Planes, submitted for publication, Urbana IL (October 1987).

19. P. C. Hammer, "Problem 2," pp. 498-499 in Proc. Symposium Pure Math vol VII: Convexity, Amer. Math. Soc. (1963).

20. R. J. Gardner and P. McMullen, “On Hammer's X-ray Problem,” J. London Math. Soc. 21 pp. 171-175 (1980). 
21. K. J. Falconer, "X-ray Problems for Point Sources," Proc. London Math Soc. (3) 46 pp. 241-262 (1983).

22. K. J. Falconer, "Hammer's X-Ray Problem and the Stable Manifold Theorem," J. London Math. Soc. (2) 28 pp. 149-160 (1983).

23. R. J. Gardner, "Symmetrals and X-rays of Planar Convex Bodies," Arch. Math 41 pp. 183-189 (1983).

24. J. A. Reeds, L. A. Shepp, P. C. Fishburn, and J. C. Lagarias, Sets Uniquely Determined by Projections, AT\&T Bell Laboratories, Murray Hill, NJ (July 1986).

25. Steven S. Skiena, "Counting the Number of $k$-Projections of a Point Set," J. Combinatorial Theory, A, (to appear).

26. P. D. Alevizos, J. D. Boissonnat, and M. Yvinec, P. D. Alevizos, J. D. Boissonnat, and M. Yvinec, P. D. Alevizos, J. D. Boissonnat, and M. Yvinec, "On the Order Induced by a Set of Rays: Application to the Probing of Non-convex Polygons," Proc. IEEE Conf. on Robotics and Automation, pp. 202-207 (1989).

27. Frank Harary and Edgar M. Palmer, Graphical Enumeration, Academic Press, New York (1973). 